\title{
Comparison of the LINC, LINC-8, and PDP-12 computers
}

RICHAKD J. CLAYTON, DIGITAL EQUIPMENT CORPORATION, Maynard, Massachusetts 01754

First, there is no one person behind any of this, and, in fact, looking at the evolution of any of the concepts that led to the classic LINC, one finds that by no means did the original LINC group immediately wash their hands of the LINC development. In fact, there has been a gradual transition to the point where Digital and other manufacturers have been more intimately involved with the evolution of the basic concepts.

The LINC-8 was done at a technical and political time, leading to the particular configuration that was generated. Since this leads to a bit of history, I'll back up for a moment. The LNNC-8 was originally envisioned as a black box that one could buy, plug onto a standard PDP 8 computer. and thereby achieve something that was program compatible with the classic LINC. That was the original concept, and, as Severo has indicated, sometimes original concepts get molded slightly in the development. We built such a machine and, in fact, ran it, and it has since been dismantled. We decided that the machine was not as good as we could accomplish. We could have essentially the same machine, running $70 \%$ faster, by removing the constraint that it be a black box plugging onto a PDP-8. This was accomplished by using the same general concepts but intermingling them a bit more closely with the PDP-8 section; that, in fact, led to the LINC-8.

The economies of production at the time that the LINC-8 was developed suggested that it have a basic PDP-8 within it. We felt an economic necessity to get the price down and to achieve the kind of

Table 1

Comparion of LINC, LINC-8 and PDP-12 Computers

These Three Systems Offer an Evolution of the LINC Concept

\begin{tabular}{|c|c|c|c|}
\hline & \multicolumn{3}{|c|}{ Major Features } \\
\hline & Classic LINC & LINC-8 & PDP-12 \\
\hline $\begin{array}{l}\text { Relative Inst. Time } \\
\text { Effective Memory } \\
\text { Scope Size (In.) } \\
\text { A-D Accuracy } \\
\text { PDP-8 Programming } \\
\text { PDP-8 Peripherals } \\
\text { Memory Expansion } \\
\text { Buffered Tape } \\
\text { Trap Capability } \\
\text { I/O } \\
\text { Teletype } \\
\text { Std Clock } \\
\text { Price } \\
\text { Price of 2nd 4K Memory }\end{array}$ & $\begin{array}{c}7 \\
2 \mathrm{~K} \\
4 \times 5 \\
8 \text { Bits } \\
\text { No } \\
\text { No } \\
? \\
\text { No } \\
\text { No } \\
\text { Direct } \\
\text { Option } \\
\text { No } \\
\text { 543K } \\
?\end{array}$ & $\begin{array}{c}1.3 \\
3 \mathrm{~K} \text { or } 4 \mathrm{~K} \\
4 \times 5 \\
9 \text { Bits } \\
\text { Yes } \\
\text { Yes } \\
32 \mathrm{~K} \\
\text { Partially } \\
\text { Yes } \\
\text { Direct/Interpret } \\
\text { Std } \\
\text { No } \\
538.5 \mathrm{~K} \\
9 \mathrm{~K} \\
\end{array}$ & $\begin{array}{c}1 \\
4 \mathrm{~K} \\
7 \mathrm{X} 9 \\
10 \mathrm{Bits} \\
\text { Yes } \\
\text { Yes } \\
32 \mathrm{~K} \\
\text { Completely } \\
\text { Yes } \\
\text { Direct } \\
\text { Std } \\
\text { Yes } \\
\$ 29.9 \mathrm{~K} \\
4.0 \mathrm{~K} \\
\end{array}$ \\
\hline
\end{tabular}

Table 2

Features Added in the Evolution of the LINC and Their Major Characteristics

1. Speed, memory size, A-D accuracy, teletype and price are all obvious.

2. PDP-8 Mode Programming:

Compatibility with 4,000 other users and developments associated with the PDP:8.

3. PDP-8 1/O Bus: Off the shelf supportable options at a reasonable price.

4. Larger Display:

When offered with half-size character and faster logic offers more extensive intercommunication.

5. Trap allows device independent software and interchange between different systems.

6. Buffered Tape: Allows continuous data collection and interchange between different systems.

7. Clock:

Allows compatibility between users and eliminates many specials.

Offers much better clock at low cost. price-performance ratio we wanted. That is where the LINC-8 came from. The LINC-8 was designed to be a machine that was either a classic LINC or a PDP-8, and that did not address itself in great detail to highly intermixed programming between the two modes or to some of the details of this intermixing. That was not a point of optimization.

Some of the most significant LINC-8 features, of course, have been the cycle time and the memory size. I think Severo had indicated, and I'm sure that most of the users concur, that 2,000 words of memory is in many cases quite confining, either for data handling or for programming. A number of users have found that the time saved in figuring out how to squeeze something into $2 \mathrm{~K}$ in many ways justifies the price for additional memory, even from $4 \mathrm{~K}$ to $8 \mathrm{~K}$. And, in the case of the current configuration of the PDP-12, that price is extremely low. In fact, well over half the PDP-12s on order are $8 \mathrm{~K}$ machines. I, personally, feel that this is because most people realize the advantage of having additional memory with which to be rather careless. This can be a real economy when it comes to the actual programming and handling of the machine. I think that is the primary reason.

There are some technical improvements, which are just fine tuning of the design, that have progressed. Specifically, they are the speeding of the display instructions, the increased accuracy of the A-to-D converter, and things of that type.

Further, the larger scope display of the PDP-12 offers many new possibilities. The buffered tape extends the usefulness of the LINC concept to real-time situations where continuous processes that require more calculations than can be done on "the fly" come in at rates faster than 2 cycles/sec. Practical, continuous, asynchronous data transfers, up to 5,000 numbers/sec, can be achieved to the tape.

The clock offered with the PDP-12 represents an extension of the LINC concept to the variable of time. Because a powerful clock is mass produced, the cost is low. When many users have totally compatible systems, the interchange of programs is far more practical and probable.

Tables 1 and 2 will serve to highlight differences among the classic LINC, LINC-8, and PDP-12. 